Neurosurg Focus 20 (4):E17, 2006

\title{
Gamma knife surgery for glioblastoma multiforme
}

\author{
R. Webster Crowley, M.D., Nader Pouratian, M.D., Ph.D., \\ AND Jason P. Sheehan, M.D., Ph.D. \\ Department of Neurological Surgery, University of Virginia Health System, Charlottesville, Virginia
}

\begin{abstract}
$\checkmark$ Despite the implementation of increasingly aggressive surgery, chemotherapy, and fractionated radiotherapy for the treatment of glioblastoma multiforme (GBM), most therapeutic regimens have resulted in only modest improvements in patient survival. Gamma knife surgery (GKS) has become an indispensable tool in the primary and adjuvant management of many intracranial pathologies, including meningiomas, pituitary tumors, and arteriovenous malformations. Although it would seem that radiosurgical techniques, which produce steep radiation dose fall-off around the target, would not be well suited to treat these infiltrative lesions, a limited number of institutional series suggest that GKS might provide a survival benefit when used as part of the comprehensive management of GBM. This may largely be attributed to the observation that tumors typically recur within a $2-\mathrm{cm}$ margin of the tumor resection cavity. Despite these encouraging results, enthusiasm for radiosurgery as a primary treatment for GBM is significantly tempered by the failure of the only randomized trial that has been conducted to yield any benefit for patients with GBM who were treated with radiosurgery. In this paper, the authors review the pathophysiological mechanisms of GKS and its applications for GBM management.
\end{abstract}

\section{KEY WORDS • glioma • glioblastoma multiforme • gamma knife surgery • radiosurgery}

$\mathrm{G}$ LIOBLASTOMA multiforme is a type of malignant glioma that comprises roughly 25 to $30 \%$ of primary brain neoplasms. ${ }^{14,19,20}$ Despite the continued development of new treatment modalities designed to target GBM, even the most aggressive treatment options that are currently available result only in modest improvements in survival. Recurrences are the rule and long-term survival the exception. Although metastatic or multicentric recurrences can happen, in up to $90 \%$ of cases, tumors typically recur within $2 \mathrm{~cm}$ of the tumor resection bed. ${ }^{8,19,24,28}$ Gamma knife surgery provides the opportunity to treat the resection bed and the surrounding margins with a high dose of radiation yet still affords a steep dose drop-off at the margin, thereby minimizing damage to the surrounding brain tissue. The efficacy of GKS for the treatment of GBM (both on initial presentation and at time of recurrence) is reviewed in this paper to ascertain the potential role of GKS in the management of these aggressive tumors.

\section{History of GKS}

The Gamma Knife was developed by Lars Leksell in the late 1960s as an alternative to open stereotactic lesion-

Abbreviations used in this paper: $\mathrm{EBRT}=$ external-beam radiotherapy; GBM = glioblastoma multiforme; GKS = gamma knife surgery; IMRT = intensity-modulated radiotherapy; KPS = Karnofsky Performance Scale; LINAC = linear accelerator; RTOG $=$ Radiation Therapy Oncology Group; SRS = stereotactic radiosurgery. ing for functional disorders. It has since become an indispensable neurosurgical tool that is used for the primary or adjuvant treatment of intracranial pathology. A type of radiosurgery, GKS differs from radiation therapy in that it is not based on variable tissue response to fractionated radiation. Instead, the Gamma Knife delivers a high dose of radiation in a single session to a stereotactically defined target by converging multiple beams of ionizing radiation. By creating a rapid radiation dose fall-off around the target, radiation can be targeted to the lesion itself, and damage to surrounding structures can be minimized (Fig. 1).

\section{Pathophysiological Considerations for GKS}

The Gamma Knife takes advantage of the natural difference in susceptibility of pathological and normal tissue. The relative radioresistance of normal brain tissue is the result of its low mitotic activity. The Gamma Knife further curtails injury to normal brain tissue by producing a rapid radiation fall-off and thus minimizes radiation exposure to adjacent normal structures. The rate at which a total dose is applied is important. A higher dose rate (that is, the same total dose applied over a shorter period of time or a larger dose in an equivalent amount of time) increases the lethality of a dose due to its greater interference with intrinsic cellular repair mechanisms during irradiation. The significance of this effect is seen most clearly at a threshold dose rate of $1 \mathrm{~Gy} /$ minute. $^{6}$ The normal tissue surrounding a target not only receives a markedly lower dose but also receives it in a lower dose rate as well. 
To understand the radiobiological effects of a single high dose of radiation on normal brain tissue, the effect of GKS on the normal parietal lobe of rats was studied. A dose of 50 Gy caused astrocytic swelling and fibrin deposition in capillary walls without changes in neuronal morphology or breakdown of the blood-brain barrier at 12 months. At $75 \mathrm{~Gy}$, more vigorous morphological changes were seen in astrocytes within 4 months. In addition, necrosis, breakdown of the blood-brain barrier, and hemispheric swelling were noted. At $120 \mathrm{~Gy}$, astrocytic swelling occurred within 1 week of irradiation, and necrosis was seen at 4 weeks, but it was not associated with hemispheric swelling. ${ }^{11}$

By delivering the radiation dose in a single session, the Gamma Knife improves the effectiveness of the target dose by 2.5 to 3 times that of the same dose delivered in a fractionated manner. Although it is generally accepted that an increase in the effectiveness of a radiation dose results in a greater rate of tumor control, little is really known about the pathophysiological mechanisms of tumor control for the Gamma Knife at the cellular level. Gamma knife-mediated tumor control is due, at least in part, to radiation-induced DNA damage. Pathohistological and imaging studies also suggest that GKS alters the microvascular supply of tumors. For example, reduced blood flow has been seen over time in meningiomas after the use of GKS. ${ }^{7}$ Early-responding tumors demonstrate the greatest reduction in blood flow. Other authors have proposed that the Gamma Knife might confer its effect by inducing apoptosis in proliferating cells. ${ }^{16,25}$ Note that the pathophysiological effect of GKS does not seem to be tumor necrosis. For necrosis, higher doses than are typically employed would be required.

Although an even dose distribution is an essential and basic concept in radiotherapy, there is some evidence that "hot spots" might be of benefit in GKS. When more than one isocenter is used to cover a target, the radiation dose distribution can become inhomogeneous. The resulting areas of local maxima are called hot spots, and they create islands of lethally injured cells that might enhance the cell kill in sublethal injury zones. Cells that are sublethally injured and are in the vicinity of lethally injured cells are more likely to undergo apoptosis than sublethally injured cells that are in the vicinity of other sublethally injured cells. Radiation geometry usually results in hot spots being located in the deep portions of the target. In tumors, this localization of hot spots might be particularly beneficial because it often corresponds to areas that receive the poorest blood supply and are therefore relatively hypoxic and less susceptible to radiation effects. ${ }^{9}$

\section{Efficacy of GKS for GBM}

The goal of GBM treatment cannot realistically be the eradication of the tumor but rather striking a balance between increasing the length of survival and maintaining an acceptable quality of life for the patient. Resection followed by EBRT is considered to be the standard treatment for GBM. ${ }^{19,23}$ Radiosurgery has been evaluated as an adjuvant treatment modality, and there has been considerable debate regarding its efficacy. However, GKS has not been evaluated as a sole treatment for GBM; all of the studies address the role of GBM as an adjuvant therapy after initial resection or at the time of tumor recurrence.

Studies involving the role of GKS in the setting of GBM have yielded conflicting results (Table 1). 10,12,14,19,24 Souhami and colleagues, ${ }^{24}$ reporting on behalf of the RTOG, were the first to conduct a multicenter randomized trial looking at the addition of SRS (including both GKS and LINAC-based radiosurgical techniques) to standard EBRT for the treatment of GBM. In this trial, SRS was used as part of the initial management of GBM in patients rather than as a salvage therapy. The researchers randomly assigned 203 patients with supratentorial GBM to receive either postoperative SRS (prescription dose 15-24 Gy) followed by EBRT and carmustine, or EBRT and carmustine without the preceding SRS. They observed no difference between the two groups of patients with regard to the primary endpoint of survival: the SRS group had a mean survival time of 13.5 months, whereas the control group had a mean survival time of 13.6 months. Although Souhami, et al., did not find a significant effect in adding SRS to the initial management of GBM, a role for GKS and other SRS techniques in the treatment of GBM should not yet be excluded, especially in light of a number of individual institution reviews that show encouraging results. ${ }^{10}$, 12,15,19 Different treatment regimens (for example, the use of GKS to provide post-EBRT boost or at time of recurrence) and treatment options for patients who have undergone gross-total resection (such patients were excluded from the study by Souhami, et al.) might prove beneficial. The rationale for such investigations is provided by numerous institutional reviews that demonstrate a life-prolonging effect of GKS in the management of GBM.

Nwokedi and associates ${ }^{19}$ performed a retrospective analysis of patients treated at the University of Maryland during a period of 6 years. Due to a shift in institutional treatment philosophy, the authors changed their practice of administering GKS at the time of tumor recurrence to giving a scheduled GKS boost within 4 weeks of the completion of EBRT. This change in practice allowed the authors to compare the two treatment paradigms using overall survival as a measurement of efficacy. They observed a nearly twofold increase in mean survival time ( 25 months compared with 13 months) in the groups of patients who received gamma knife treatment as a planned boost within 4 weeks of EBRT. This treatment paradigm differs from that of Souhami and coworkers, who administered SRS 1 week prior to the initiation of EBRT.

Other studies have further supported the role of radiosurgery as adjuvant therapy in GBM. Kondziolka, et al., ${ }^{12}$ compared their series of patients with GBM who were treated by GKS with historical control groups. Again, in contrast to the study by Souhami and associates, ${ }^{24}$ in this series patients generally received GKS 5 to 8 months after initial diagnosis (and after other therapies had been completed, including EBRT and chemotherapy) or at the time of recurrence. Patients who received GKS (mean prescription dose of $15.2 \mathrm{~Gy}$ ) as part of the initial treatment regimen had an increased median survival of 20 months compared with 11.2 months in the control group. The researchers also reported a median survival of 30 months after GKS for those patients who were treated with GKS at the time of tumor progression. Loeffler, et al. ${ }^{15}$ treated patients with radiosurgery (prescription doses rang- 
TABLE 1

Literature review of studies involving the role of GKS in the setting of GBM*

\begin{tabular}{|c|c|c|c|c|c|}
\hline Authors \& Year & $\begin{array}{l}\text { Form of Stereotactic } \\
\text { Radiotherapy }\end{array}$ & Timing of SRS $\dagger$ & $\begin{array}{c}\text { No. of GBM } \\
\text { Cases Analyzed }\end{array}$ & $\begin{array}{l}\text { Prescription } \\
\text { Dose (Gy) }\end{array}$ & $\begin{array}{c}\text { Median } \\
\text { Survival (mos) }\end{array}$ \\
\hline Loeffler, et al., 1992 & gamma knife & initial & 23 & $12-15$ & 26 \\
\hline Kondziolka, et al., 1997 & gamma knife & initial, recurrence & 64 & 15.5 & 26 \\
\hline Shrieve, et al., 1999 & LINAC & initial & 60 & $6-24$ & 19.9 \\
\hline Nwokedi, et al., 2002 & gamma knife & initial & 64 & 17.1 & 25 \\
\hline Voynov, et al., 2002 & IMRT & recurrence & 4 & 30 & 10.1 \\
\hline Wang, et al., 2002 & LINAC & initial & 30 & 19.4 & 13 \\
\hline Floyd, et al., 2004 & IMRT & initial & 20 & 50 & 7 \\
\hline Souhami, et al., 2004 & gamma knife \& LINAC & initial & 186 & $15-24$ & 13.5 \\
\hline Combs, et al., 2005 & FSRT & recurrence & 59 & 36 & 21 \\
\hline Combs, et al., 2005 & gamma knife & recurrence & 32 & 15 & 22 \\
\hline Hsieh, et al., 2005 & gamma knife & initial, recurrence & 51 & 12 & 14.3 \\
\hline Narayana, et al., 2006 & IMRT & initial & 41 & 59.4 & 9 \\
\hline
\end{tabular}

* FSRT $=$ fractionated stereotactic radiotherapy.

$\dagger$ As part of initial treatment compared with time of tumor recurrence.

ing from 10-20 Gy) 2 to 4 weeks after EBRT, which was similar to the treatment provided by Nwokedi and colleagues, ${ }^{19}$ and they also observed a median overall survival rate of 26 months for those patients. Reports demonstrate that GKS particularly benefits patients with a KPS ranking of at least 90 and those patients who have received adjuvant chemotherapy. ${ }^{10}$ At the University of Virginia, our unpublished experience of 56 patients appears to support the use of GKS for GBM, providing a small survival advantage. Although the results of these institutional reviews present encouraging data, the patients receiving GKS in each series usually represent a highly selective group; in none of the series were all the patients at an institution treated with GKS. Therefore, one must take caution in interpreting each institution's results and realize that such results cannot necessarily be generalized.

From a theoretical standpoint, it is difficult to understand how a highly invasive tumor, such as a GBM, can be treated effectively with such a focused treatment as GKS. However, GKS can be used to treat the largest concentration of the residual tumor or the resection cavity, based on neuroimaging studies. It seems clear that no single treatment modality in the neurooncology armamentarium is a magic bullet for such tumors, and as such, this multimodality approach (that is, GKS, EBRT, cytoreductive surgery, and chemotherapy) to GBM might be prudent.

\section{Other Forms of Stereotactic Radiosurgery and Radiotherapy}

Most studies of SRS for GBM have used GKS as the primary treatment modality. Other SRS techniques, however, might also be useful and beneficial for the treatment of high-grade gliomas (Table 1). The utility of LINACbased radiosurgery also has been reported. ${ }^{22,24,29}$ Souhami and colleagues ${ }^{24}$ did not distinguish between patients treated with LINAC-based radiosurgery and those treated with GKS, whereas Shrieve, et al., ${ }^{22}$ exclusively used the LINAC system. They reported that the LINAC system might provide a survival benefit to selected patients with GBM, and identified two important prognostic factors: age and RTOG classification. Although patients younger than 40 years of age had a median survival of 49 months, patients older than 40 years of age had a median survival of 18.2 months $(\mathrm{p}<0.001)$. Moreover, patients with RTOG Class III tumors had a significantly longer median survival than those with RTOG Classes IV and V tumors (29.5 months compared with 19.2 and 18.2 months, respectively). Other than the patients included in the report by Souhami, et al., further significant studies of LINACbased radiosurgery have not been reported.

The potential role of hypofractionated radiotherapy that uses LINAC technology, such as IMRT in the management of GBM, has also been investigated. Intensity-modulated radiotherapy is designed to deliver a relatively high dose of radiation to targeted lesions but spare adjacent normal tissue in a fractionated manner., ${ }^{5,18,26}$ Voynov, et al. ${ }^{26}$ reported a median survival of 10 months when IMRT was used at the time of recurrence in patients with highgrade gliomas. Also, IMRT has been investigated as an alternative to EBRT but was found to have limited utility. Floyd and associates ${ }^{5}$ reported no improvement in time to progression or length of survival when IMRT was used in place of EBRT. Instead, they found an increased need for surgery due to necrosis. Narayana, et al., ${ }^{18}$ reported similar findings in a series of patients who were treated with IMRT in place of EBRT. The authors suggested that although IMRT does not improve overall survival, it might be advantageous in some scenarios because of the decreased time required for administration of radiation..$^{5,18}$

Combs and coworkers ${ }^{2}$ reported on their experience using fractionated stereotactic radiotherapy at the time of tumor recurrence in 59 patients with GBM. They retrospectively reported a median overall survival of 21 months after primary diagnosis and of 8 months after treatment with fractionated stereotactic radiotherapy in patients with GBM. They suggest that fractionated stereotactic radiotherapy is well tolerated and might be particularly appropriate for patients with large tumors because the risk of radiation-induced side effects increases with treatment volumes. This has not been evaluated in a randomized clinical trial to date.

\section{Complications}

Like other neurosurgical procedures, GKS has associat- 


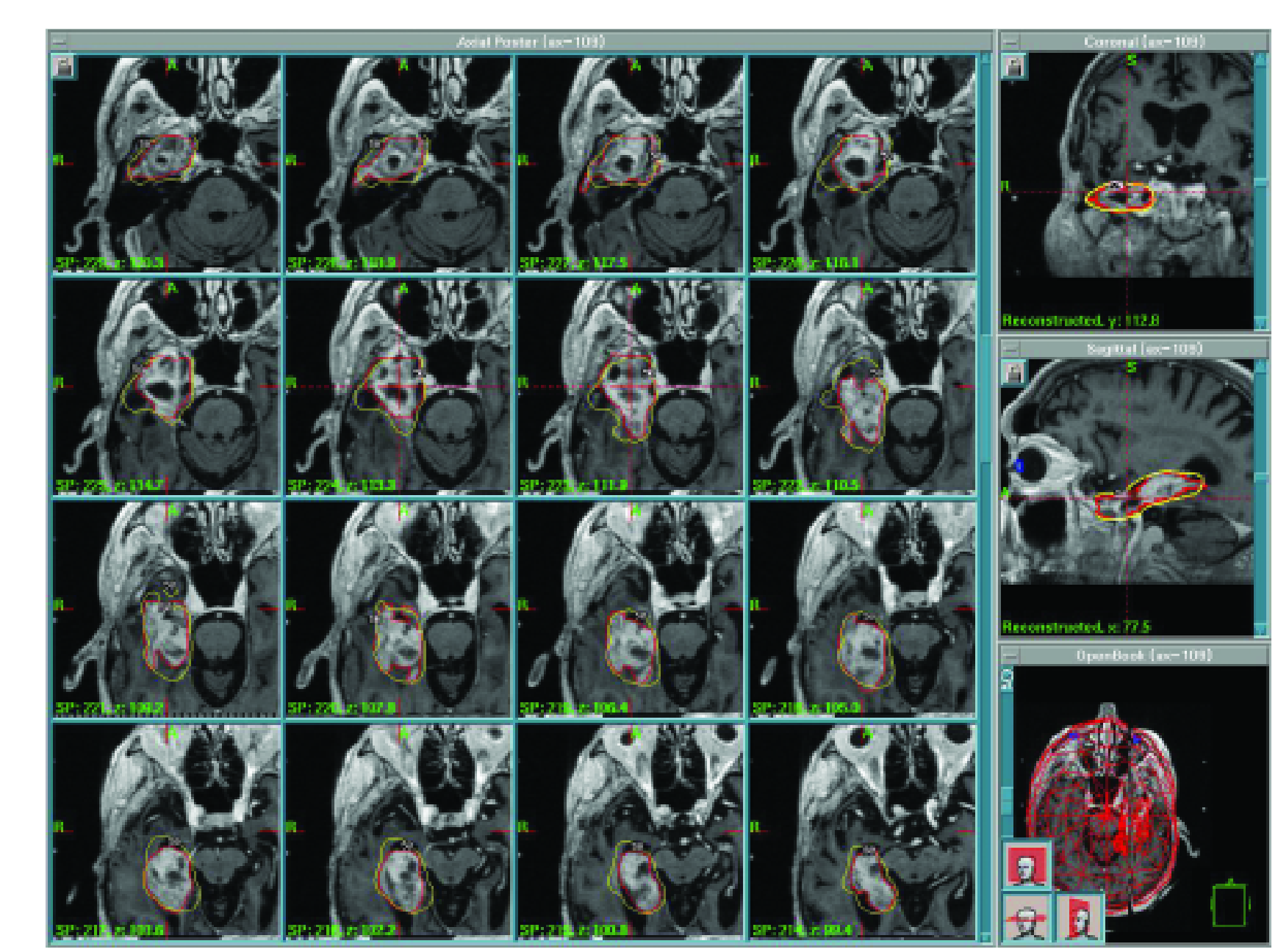

FIG. 1. Gamma knife treatment plan for a 71-year-old man after initial resection, fractionated radiotherapy, and chemotherapy. The patient had a KPS ranking of 90. In this instance, GKS was used to treat recurrent disease. The lines within the treatment plan represent isodose lines and illustrate the steep radiation dose fall-off (yellow lines) at the margins of the treatment area (red lines).

ed risks and the potential for complications. The limited life expectancy of patients with GBM, however, limits the chance for long-term complications (such as new tumor induction) that are occasionally observed with GKS treatment of patients who have benign tumors. Nonetheless, radiation toxicity and parenchymal injury associated with radiosurgery have been observed. Radiation necrosis is one such complication that can be seen with radiosurgery. With radiotherapy, increases in radiation dose can increase mean survival time, ${ }^{1,27}$ and the rate of radiation necrosis seen with radiotherapy has similarly been linked to the amount of radiation given. ${ }^{17}$ Although radiation necrosis remains a potential complication of treatment, SRS has allowed the treating physician to both maximize the local administration of radiation and limit the rate of toxicity to normal brain tissue. Kondziolka and colleagues ${ }^{12}$ observed this in $1.6 \%$ of their patients with GBM, although other series have shown varying numbers. ${ }^{3,10}$ Other potential complications that have been observed in association with GKS include cranial neuropathies, vascular injuries (including, for example, carotid artery stenosis), posttreatment edema, and induction of new tumors., ${ }^{4,21}$ Cranial neuropathies and vascular injuries are rarely seen as a result of GBM treatment because the lesions are not typically close to large vascular structures or cranial nerves. Posttreatment edema remains a potential complication, but it has not been noted as a significant problem in the literature. ${ }^{10,19,24}$ In general, when it occurs, patients complain of severe headache and often can be managed effectively with administration of oral corticosteroids. The mechanism of edema formation is unclear, but symptoms generally occur 1 to 6 months after radiosurgery.

\section{Limitations}

As with almost any treatment option for these lesions, the role of GKS in treating GBM is limited. Many of the series dealing with the treatment of GBM use tumor size as a selection criterion for radiosurgical eligibility, with 35 to $40 \mathrm{~mm}$ being the maximum acceptable tumor size. ${ }^{12,19}$ Tumor debulking is therefore especially necessary in patients with larger tumors, although it is advocated in nearly all cases of GBM regardless of tumor volume. Other selection criteria that have been applied include having a 
KPS ranking greater than 50, a life expectancy longer than 3 months, a histopathological diagnosis of GBM, and an age less than 75 years. ${ }^{10,12}$ One limitation inherent to gamma knife SRS is that it delivers radiation over a single session, and thus it does not encounter multiple cell cycles or mitotic phases. It has been argued, however, that the radiation effect that is seen with a single session of GKS is sufficient for achieving adequate tumor cell death. ${ }^{12}$

\section{Conclusions}

Despite ongoing developments in the field of neurooncology, the treatment of GBM remains a challenging and frustrating problem for neurosurgeons. Contributing to this frustration is the knowledge that the disease is highly aggressive and infiltrative despite a seemingly discrete enhancing tumor margin on magnetic resonance images. Several institutional series suggest that GKS might have potential benefit when administered after the completion of EBRT or chemotherapy as part of the initial treatment regimen or at the time of recurrence or progression. Although these results are encouraging, enthusiasm for radiosurgery as a primary treatment for GBM is significantly tempered by the failure of the only randomized trial to date to show any benefit for patients with GBM who were treated with radiosurgery. ${ }^{24}$ Despite the failure of this trial, further randomized studies might be indicated to investigate whether administering radiosurgery at different times in the management of patients with GBM (for example, after completion of EBRT or chemotherapy) or to those in select subpopulations (for example, basing selection on age or RTOG classification) could provide any appreciable benefit to patients.

\section{References}

1. Bleehen NM, Stenning SP: A Medical Research Council trial of two radiotherapy doses in the treatment of grades 3 and 4 astrocytoma. The Medical Research Council Brain Tumour Working Party. Br J Cancer 64:769-774, 1991

2. Combs SE, Thilmann C, Edler L, et al: Efficacy of fractionated stereotactic reirradiation in recurrent gliomas: long-term results in 172 patients treated in a single institution. J Clin Oncol 23: 8863-8869, 2005

3. Combs SE, Widmer V, Thilmann C, et al: Stereotactic radiosurgery (SRS): treatment option for recurrent glioblastoma multiforme (GBM). Cancer 104:2168-2173, 2005

4. DiBiase SJ, Kwok Y, Yovino S, et al: Factors predicting local tumor control after gamma knife stereotactic radiosurgery for benign intracranial meningiomas. Int J Radiat Oncol Biol Phys 60:1515-1519, 2004

5. Floyd NS, Woo SY, Teh BS, et al: Hypofractionated intensitymodulated radiotherapy for primary glioblastoma multiforme. Int J Radiat Oncol Biol Phys 58:721-726, 2004

6. Hall EJ, Marchese M, Hei TK, et al: Radiation response characteristics of human cells in vitro. Radiat Res 114:415-424, 1988

7. Hawighorst H, Engenhart R, Knopp MV, et al: Intracranial meningeomas: time-and dose-dependent effects of irradiation on tumor microcirculation monitored by dynamic MR imaging. Magn Reson Imaging 15:423-432, 1997

8. Hochberg FH, Pruitt A: Assumptions in the radiotherapy of glioblastoma. Neurology 30:907-911, 1980

9. Hopewell JW, Wright EA: The nature of latent cerebral irradiation damage and its modification by hypertension. Br J Radiol 43:161-167, 1970

10. Hsieh PC, Chandler JP, Bhangoo S, et al: Adjuvant gamma knife stereotactic radiosurgery at the time of tumor progression potentially improves survival for patients with glioblastoma multiforme. Neurosurgery 57:684-692, 2005
11. Kamiryo T, Kassell NF, Thai QA, et al: Histological changes in the normal rat brain after gamma irradiation. Acta Neurochir (Wien) 138:451-459, 1996

12. Kondziolka D, Flickinger JC, Bissonette DJ, et al: Survival benefit of stereotactic radiosurgery for patients with malignant glial neoplasms. Neurosurgery 41:776-785, 1997

13. Kondziolka D, Levy EI, Niranjan A, et al: Long-term outcomes after meningioma radiosurgery: physician and patient perspectives. J Neurosurg 91:44-50, 1999

14. Lederman G, Wronski M, Arbit E, et al: Treatment of recurrent glioblastoma multiforme using fractionated stereotactic radiosurgery and concurrent paclitaxel. Am J Clin Oncol 23:155-159, 2000

15. Loeffler JS, Alexander E III, Shea WM, et al: Radiosurgery as part of the initial management of patients with malignant gliomas. J Clin Oncol 10:1379-1385, 1992

16. Mareková M, Cáp J, Vokurková D, et al: Effect of therapeutic doses of ionising radiation on the somatomammotroph pituitary cell line, GH3. Endocr J 50:621-628, 2003

17. Marks JE, Baglan RJ, Prassad SC, et al: Cerebral radionecrosis: incidence and risk in relation to dose, time, fractionation and volume. Int J Radiat Oncol Biol Phys 7:243-252, 1981

18. Narayana A, Yamada J, Berry S, et al: Intensity-modulated radiotherapy in high-grade gliomas: clinical and dosimetric results. Int J Radiat Oncol Biol Phys 64:892-897, 2006

19. Nwokedi EC, DiBiase SJ, Jabbour S, et al: Gamma knife stereotactic radiosurgery for patients with glioblastoma multiforme. Neurosurgery 50:41-47, 2002

20. Polednak AP, Flannery JT: Brain, other central nervous system, and eye cancer. Cancer 75:330-337, 1995

21. Pollock BE: Stereotactic radiosurgery for intracranial meningiomas: indications and results. Neurosurg Focus 14(5):E4, 2003

22. Shrieve DC, Alexander E III, Black PM, et al: Treatment of patients with primary glioblastoma multiforme with standard postoperative radiotherapy and radiosurgical boost: prognostic factors and long-term outcome. J Neurosurg 90:72-77, 1999

23. Simpson JR, Horton J, Scott C, et al: Influence of location and extent of surgical resection on survival of patients with glioblastoma multiforme: results of three consecutive Radiation Therapy Oncology Group (RTOG) clinical trials. Int J Radiat Oncol Biol Phys 26:239-244, 1993

24. Souhami L, Seiferheld W, Brachman D, et al: Randomized comparison of stereotactic radiosurgery followed by conventional radiotherapy with carmustine to conventional radiotherapy with carmustine for patients with glioblastoma multiforme: report of Radiation Therapy Oncology Group 93-05 protocol. Int J Radiat Oncol Biol Phys 60:853-860, 2004

25. Tsuzuki T, Tsunoda S, Sakaki T, et al: Tumor cell proliferation and apoptosis associated with the Gamma Knife effect. Stereotact Funct Neurosurg 66 Suppl 1:39-48, 1996

26. Voynov G, Kaufman S, Hong T, et al: Treatment of recurrent malignant gliomas with stereotactic intensity modulated radiation therapy. Am J Clin Oncol 25:606-611, 2002

27. Walker MD, Strike TA, Sheline GE: An analysis of dose-effect relationship in the radiotherapy of malignant gliomas. Int $\mathbf{J}$ Radiat Oncol Biol Phys 5:1725-1731, 1979

28. Wallner KE, Galicich JH, Krol G, et al: Patterns of failure following treatment for glioblastoma multiforme and anaplastic astrocytoma. Int J Radiat Oncol Biol Phys 16:1405-1409, 1989

29. Wang YY, Bao XF, Li SY, et al: [Prognostic factors for deep situated malignant gliomas treated with linac radiosurgery.] Ai Zheng 21:1149-1153, 2002 (Chn)

Manuscript received January 20, 2006.

Accepted in final form March 16, 2006.

Address reprint requests to: Jason P. Sheehan, M.D., Ph.D., Box 800-212, Department of Neurological Surgery, University of Virginia Health System, Charlottesville, Virginia 22908-0212. email: jps2f@hscmail.mcc.virginia.edu. 